\title{
Correction to: Foodscapes of Contemporary Japanese Women Writers \\ Masami Yuki
}

Author name is incorrectly captured in all chapters of this book. The correct author name should be Masami Yuki. This has been corrected.

The updated online version of the original book can be found at https://doi.org/10.1057/9781137477231.

(C) Masami Yuki, translated by Michael Berman, 2020

M. Yuki, Foodscapes of Contemporary Japanese Women Writers, 